\title{
VI. BIBLIOGRAPHY
}

\section{A. Primary Works}

\section{1) Ödön von Horváth}

Gesammelte Werke. 8 vols. Hg. Traugott Krlschke und Dieter H1ldebrandt. Frankfurt/M., Suhrkamp, 1972. (werkausgabe edition suhrkamp).

Kaslmir und Karoline Volksstück. Hg. Traugott Krischke. Frankfurt/M., 1972. (Bibliothek Suhrkamp 316). Contains variants.

Glaube Llebe Hoffnung Eln klelner Totentanz. Hg. Traugott Krischke. Frankfurt/M., 1973. (Bibliothek Suhrkamp 361). Contains variants.

Italienische Nacht Volksstück. Hg. Traugott Krischke. Frankfurt/M., 1974. (Bibliothek Suhrkamp 410). Contains Das Wochenendspiel and variants.

Geschichten aus dem Wiener Wald. Volksstück in drel Tellen. Frankfurt/M., 1977. (Bibliothek Suhrkamp 247). Enlarged new edition of Bibliothek Suhrkamp 247, 1970. Includes the version "in sieben Bildern" as well as varlants and a 'Nacherzählung' by Peter Handke.

2) Other Authors

Beaumarcha1s, Pierre Augustin Caron de. Thêâtre Complet. Ed. Maurice Allem/Paul Courant. Paris, Librairie Gallimard, 1957. (Bibliothèque de la Plélade).

Brecht, Bertolt. Gesammelte Werke in 20 Bänden. Frankfurt/M., Suhrkamp, $196 \overline{7 .}$

Brecht, Bertolt. Schriften zum Theater. Frankfurt/M., Suhrkamp, 1957. (BS 41).

Hasenclever, Walter. Gedichte, Dramen Prosa. Hg. Kurt Pinthus. Reinbek be1 Hamburg, RowohIt, $196 \overline{3 .}$ 
Hauptmann, Gerhart. Der Biberpelz. Edition und Nachwort von Hubert Razinger. Berlin, Ullstein, 1959. (Propyläen Textausgabe).

Ka1ser, Georg. Nebeneinander. EIn Volksstück von 1923. Stuttgart, Reclam, 1978.

Ka1ser, Georg. Werke In dre1 Bänden. Hg. Klaus Kändler. Berlin/Welmar, Auf bau verlag, 1979 .

Motte-Fouqué, Friedrich de la. Undine. Elne Erzählung. LeIpzig, Reclam, 1937.

Mus11, Robert. Vinzenz und die Freundin bedeutender Männer. Posse in dret Akten (1923). Robert Musil. Gesammelte Werke, $\overline{\mathrm{Bd.6}}, \overline{\mathrm{Hg}}$. Adolf Frisé. Relnbek bel Hamburg, Rowohlt, 1978, pp.409-452.

Musil, Robert. Tagebücher Aphorlsmen Essays und Reden. Hg. Adolf Frisé. Reinbek bel Hamburg, Rowohlt, 1955.

Paul1, Hertha. 'L'Inconnue de la Seine'. Berliner Tagblatt. $\mathrm{Nr} .521,4$ November, 1931.

Nietzsche, Friedrich. Aus dem NachlaB der Achtziger Jahre. Nletzsche, Werke IV. $\overline{\text { Hg. }}$ Karl Schlechta. Frankfurt/M., Berlin, Wien, Uilstein, 1969.

Plautus. Die Komödien des Plautus. Bd.3. Ubersetzt von Ludwig Gurlitt. Berlin, Propyläen Verlag, 1922.

Schiller, Frledrlch. Gesamtausgabe in 20 Bänden. München, dtv. 1966.

Schönherr, Karl. Gesammelte Werke. Hg. Vincent Chiavacc1. Wien, Donau Verlag, 1948.

Wedekind, Frank. Prosa Dramen Verse. München, Langen-Mïlller, n.d.

Zuckmayer, Carl. Der fröhliche Welnberg. In: Günter Rühle. Zeit und Theater 1925-1933. Bd.3: Von der Republik zur Diktatur. Frankfurt/M., Berlin, Wien, Uilstein, 1972. 


\section{B. Secondary Literature}

\section{1) On Odön von Horváth}

Arntzen, Helmut. 'Horváth Geschichten aus dem Wiener Wald'. In: Die deutsche Komödie. Vom Mittelalter bis zur Gegenwart. Hg. Walter Hinck. Dísseldorf, August Bagel Verlag, 1977, pp. 246-268.

Ballin, Dolly Elisabeth. Irony in the dramatic work of Ödön von Horváth. Ph.D Univ. of Washington, 1969.

Bance, A.F. 'Ödön von Horváth: Kasimir und Karoline'. In: Austrian Life and Literature 1780-1938. Eight Essays edited by Peter Branscombe. Edinburgh, Scottish Academlc Press, 1978, pp.81-93.

Bartsch, Kurt/Baur, Uwe/Goltschnigg, Dietmar (Hg.). Horváth-Diskussion. Kronberg/Ts., Scriptor, 1976. (Monographien Literaturwissenschaft 28 ).

Bauer, Roger. 'Reflexionen über Ödön von Horváth und das Wiener Volksstück'. In: Roger Bauer. Utopla und Welterfahrung: Stefan Andres und sein Werk im Gedächtnis seiner Freunde. Mïnchen, P1per, 1972 , pp.236-242.

Baur, Uwe. 'Sport und Literatur in den Zwanziger Jahren. Horváths Sportmärchen und dle Münchener Nonsense-Dichtung'. In: Kurt Bartsch/Uwe Baur/Dietmar Goltschnigg ( $\mathrm{Hg}_{\circ}$ ). Horváth-Diskussion. Kronberg/Ts., Scriptor, 1976. (Monographien Literaturwissenschaft 28), pp.138-156.

Berczik, Árpád. 'Ödön von Horváth und Kálmán Mikszáth'. Arbeiten zur deutschen Philologie, 7 (1973), pp.61-82.

Bruns, Dirk. 'Horvath's revival of the folk play and the decline of the Welmar Republic'. new german critique, 18 (1979), pp. 107-135.

Carl, Rolf-Peter. 'Theatertheorle und Volksstïck bel Ödön von Horváth'. In: Jürgen Hein (Hg.). Theater und Gesellschaft. Das Volksstück im 19. und 20. Jahrhundert. Düsseldorf,

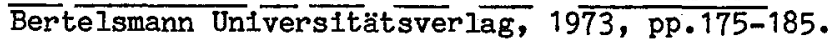

Cyron-Hawryluk, Dorota. Zeltgenössische Problematik in den Dramen Ödön von Horváths. Wroclaw, 1974. (Acta Universitatis Wratislaviensis, Nr.209). 
Doppler, Alfred. 'Ödön von Horváth: Geschlchten aus dem Wiener Wald'. In: Das österrelchische Volksstück. Hg. Inst1tut für Österrelchkunde. Wien, Ferdinand Hirt, 1971, pp.77-92.

Doppler, Alfred. 'Bemerkungen zur dramatischen Form der Volksstuicke Horváths'. In: Kurt Bartsch/Uwe Baur/Dletmar Goltschn1gg (Hg.). Horváth-Diskussion. Kronberg/Ts., Scriptor, 1976. (Monographlen Literaturwissenschaft 28), pp.11-21.

Emrich, Wilhelm. 'Die Dummhe1t oder das Gefühl der Unendlichke1t: Ödön von Horváths Kritik'. In: Wilhelm Emrich. Gelst und W1dergelst. Frankfurt/M., Athenäum, 1965.

Erken, Günter. 'Ödön von Horváths Geschlchten aus dem Wiener Wald'. In: Von Lessing bis Kroetz EInführung In die Dramenanalyse. Kursmodelle und sozialgeschichtliche Materialien für den Unterricht. Hg. Jan Berg et.al. Kronberg/Ts., Scriptor verlag, 1975, pp.138-179.

Frangois, Jean-Claude. Histoire et fletion dans le théâtre d'Üdön von Horváth. Grenoble, Presses UnIversitaIres de Grenoble, 1978 .

Frangols, Jean-Claude.' 'Brecht, Horváth and the Popular Theatre'. Trans. by Ellsabeth Ann Brown. new german critique, 18 (1979), pp.136-150. First published In $\underline{\text { Revue }}$ d'Allemagne, 11 (1979), pp.44-61.

Fritz, Axel. Ödön von Horváth als Kritiker selner Zeit. Studien zum Werk und zu selnem Verhalten zum politischen, sozlalen und kulturellen Zeitgeschehen. München, LIst, 1973. (List Taschenbuch der Wissenschaft, 1446).

Fritz, Axel. 'Ödön von Horváth als Kritiker seiner Zeit'. In: Traugott Krlschke/Dieter Hildebrandt (Hg.). Uber Ödön von Horváth. Frankfurt/M., Suhrkamp, 1972 (es 584), pp. 46-58.

Gamper, Herbert.' 'Horváth und dle Folgen. Das Volksstilck? tber neue Tendenzen im Drama'. Sonderheft Theater Heute, 1971, pp.73-77.

Gamper, Herbert. 'Die Zelchen des Todes und des Lebens. Zu bisher kaum beachteten Konstruktionselementen in Horváths vier "Fräuleinstücken"'. Theater Heute 15 (1974) Nr.3, pp. 1-6. 
Gamper, Herbert. Zur schönen Aussicht. Vorbereitende Überlegungen zum stück. Stuttgart, württembergische

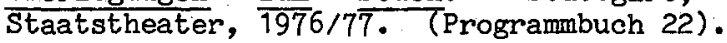

Gamper, Herbert. 'Todesbilder in Horváths Werk'. In: Kurt Bartsch/Uwe Baur/Dietmar Goltschnigg (Hg.). Horváth-Diskussion. Kronberg/Ts., Scriptor, 1976. (Monographien Literaturwissenschaft 28), pp.67-81.

Genno, Charies N. 'KItsch" elements in Horváth's Geschichten aus dem Wiener Wald'. German Quarterly, 45 (1972), pp.311-323. W L L

Günther, Gisela. Die Rezeption des dramatischen Werkes von Ödön von Horváth von den Anfängen bis 1977. Diss. Un1v. GöttIngen, $197 \overline{8}$

Haag, Ingrid. 'Ödön von Horváth und die "Monströse Idylle"' Recherches Germaniques 6 (1976), pp.152-168.

Haag, Ingrid. 'Das traurige "Happy-End". Zur Struktur der "FräuleInstiucke" Ödön von Horváths'. Austriaca 6 (1978), pp.169-186.

Haag, Ingrid. 'Zu Horváths "Komödie des Menschen"'. Austriaca 14, (1982), pp.169-186.

Hein, Jürgen. 'Ödön von Horváth: Kasimir und Karoline.' In: Deutsche Dramen. Interpretationen zu Werken von der Aufklärung bis zur Gegenwart. Bd.2. $\overline{\mathrm{H} g}$. Harro Müller-Michaels. Königstein/Ts., Athenäum, 1981, pp.42-67.

Herschbach, Robert Alexander. The 'Volksstück' of Ödön von Horváth: Text and Context. Th.D. Unfv. of Massachusetts, 1973.

Herschbach, Robert Alexander. 'Horváth's plays in exile: From desperation to "Wunschtraum"'. In: Deutsches ExIldrama und Exilliteratur. Hg. Wolfgang Elfe/James Hardin/Günther Holst. Bern, Peter Lang, 1977, pp.127-132. (Akten des Exilliteratur-Symposiums der University of South Carolina, 1976).

Hildebrandt, Dieter. $\frac{\text { Horváth }}{\text { Bilddokumenten. }}$ in $\frac{\text { Selbstzeugnissen }}{\text { inbek }}$ bel Hamburg, Rowohlt, 1975. (rororo bildmonographien 231). 
H1llach, Ansgar. 'Das Volksstilck als Kosmologie der Gewalt. Pyschologie in Ödön von Horváths Revolte auf Cote 3018'. Germanisch-Romanische Monatsschrift 12 (1974), pp.223-243.

Himmel, Hellmuth. 'Ödön von Horváth und die Volksstücktradition'. In: Traugott Krischke (Hg.). Ödön von Horváth. Frankfurt/M., Suhrkamp, 1981 (st 2005), pp. $\overline{46-56 .}$

Huder, Walter. 'Inflation als Phänomen der Existenz'. In: Traugott Krischke (Hg.). Materlalien zu Ödön von Horváth. Frankfurt/M., Suhrkamp, 1970 (es 436), pp.173-179.

Huder, Walter. 'Ödön von Horváth. Existenz und Produktion im Exil'. In: Die deutsche Exilliteratur. Hg. Manfred Durzak. Stuttgart, Ph1lipp Reclam jun., 1973, pp.232-244.

Hummel, RIchard. DIe Volksstiucke Ödön von Horváths. Baden-Baden, Johannes Hertel Verlag, 1970.

Huish, Ian. Horváth. A Study. London, Heinemann, 1980.

Jarka, Horst. 'Ödön von Horváth und das Kitschige'. Zeitschrift fur deutsche Philologie 91 (1972), pp.558-585.

Joas, Hans. 'Ödön von Horváth: Kasimir und Karoline'. In: Traugott Kr1schke (Hg.). Materialien zu Odön von von Horváths 'Kasimir und Karoline'. Frankfurt/M., Suhrkamp, 1973 (es 611), pp.47-62.

Kahl, Kurt. Ödön von Horváth. Velber, Friedrich, 1966. (Friedrichs Dramatiker des Welt-Theaters 18 ).

Kahl, Kurt. 'Der Dramatiker der Krise'. In: Traugott Krischke (Hg.). Materialien zu Ödön von Horváth. Frankfurt/M., Suhrkamp, 1970 (es 436), pp.147-161.

Karasek, Helmuth. 'Kasimir und Karoline'. In: Traugott Krischke (Hg.). Materialien zu Ödön von von Horváths 'Kasimir und Karoline'. Frankfurt/M., Suhrkamp, 1973 (es 611), $\mathrm{pp} \cdot \overline{63-76 .}$

Klenzle, Siegfried. Ödön von Horváth. Berlin, Colloquium Verlag, 1977. (Köpfe des Xx. Jahrhunderts, Bd.87).

Klotz, Volker. Dramaturgie des Publikums. Wle Bühne und Publikum aufelnander eingehen bei RaImund, Büchner, Wedekind, Horváth, Gatti und im politischen Agitationstheater. München, Carl Hanser verlag, 1976. 
Krammer, Jenö. Ödön von Horváth Leben und Werk aus ungarischer Sicht. Wien, Wissenschaftiche Buchreine der internationalen Lenau-Gesellschaft, 1969.

Krischke, Traugott (Hg.). Materialien zu Ödön von Horváth. Frankfurt/M., Suhrkamp, 1970 (es 436).

Krischke, Traugott/Hildebrandt, Dieter (Hg.). Über Ödön von Horváth. Frankfurt/M., Suhrkamp, 1972 (es 584).

Kr1schke, Traugott (Hg.). Ödön von Horváth. Frankfurt/M., Suhrkamp, 1981. (st 2005).

Krischke, Traugott (Hg.). Materialien zu Ödön von Horváths Geschichten aus dem Wiener Wald. Frankfurt/M., suhrkamp, 1972 (es 533).

Krischke, Traugott (Hg.). Materialien zu Ödön von Horváths 'Kasimir und Karoline'. Frankfurt/M., Suhrkamp, $\overline{1973 \text { (es }}$ 611).

Krischke, Traugott (Hg.). Materialien zu Ödön von Horváths 'Glaube Llebe Hoffnung'. Frankfurt/M., Suhrkamp, 1973 (es 671).

Krischke, Traugott (Hg.). Ödön von Horváth Glaube Llebe Hoffnung. Edition und Nachwort von Traugott Krischke. Frankfurt/M., Suhrkamp, 1973 (BS 361).

Krlschke, Traugott ( $\left.\mathrm{Hg}_{*}\right)$. Ödön von Horváth Italienische Nacht. Edition und Nachwort von Traugott Krischke. Frankfurt/M., Suhrkamp, 1974 (BS 410).

Krischke, Traugott (Hg.). Ödön von Horváth Geschichten aus dem Wiener Wald. Edition und Nachwort von Traugott Krischke. Frankfurt/M., Suhrkamp, 1977 (BS 247).

Krischke, Traugott. Ödön von Horváth Kind seiner Ze1t. München, Heyne, 1980.

Krischke, Traugott/Prokop, Hans. Ödön von Horváth. Leben und Werk in Daten und Bildem. Frankfurt/M., Insel, 1977. (Insel taschenbuch 237).

Kurzenberger, Hajo. Horváths Volksstücke: Beschrelbung eines poetischen Verfahrens. Minchen, Wilhelm Fink, 1974. 
Lindken, Hans-Ulrich. 'Illusion und Wirklichkelt in Ödön von Horváths Geschichten aus dem Wiener Wald' - Modern Austrian Literature $9(1976), \mathrm{pp} .26-43$.

Matuszczak, Joanna Barbara. Asoziale Flguren $\frac{\text { Im }}{\text { New }} \frac{\text { Werk }}{\text { York, Ödön }} \frac{\text { von }}{1977 .}$
Horváth. Ph.D. Univ.

Melzer, Gerhard. Das Phänomen des Tragikomischen. Untersuchungen zum Werk von Karl Kraus und Ödön von Horváth. Kronberg/Ts., Scriptor Verlag, 1976.

Mennemeler, Franz Norbert. 'Bürgerlicher Realismus am Vorabend der Naz1-Herrschaft (Ödön von Horváth)'. In: Franz Norbert Mennemeler. Modernes $\frac{\text { Deutsches }}{\text { Gegenwart. }}$ München, Wilhelm Fink, $\frac{2 .}{1975}, \frac{1933}{\text { pp.12-41. }}$ bis zur

Miller, Karl. 'Ödön von Horváths Weg nach Innen'. Österreich in Geschichte und Literatur mit Geographie 26,5 (1982), pp.284-297.

Nolting, Winfried. Der totale Jargon. München, Wilhelm Fink, 1976.

Pollow, Helmut. 'Ödön von Horváth - zum Beispiel. Zur Rezeption von Dramatik der zwanziger Jahre'. Weimarer Beiträge 6 (1979), pp.143-153.

Pichler, Meinrad. 'Von Aufstelgern und Deklassierten: Ödön von Horváths I1terarische Analyse des Klelnbürgertums und thr Verhältnis zu den Aussagen der historischen Sozialwissenschaften'. In: Österrelchische Literatur seit den zwanzlger Jahren. Hg. Friedbert Aspetsberger. Bundesverlag, 1979, pp.55-67. Also In: Traugott Krischke (Hg.). Ödön von Horváth. Frankfurt/M., Suhrkamp, 1981 (st 2005), $\overline{p p .67}-\overline{86}$.

Relnhardt, Hartmut. "Demaskierung" als moralische Provokation. Beobachtungen am dramatischen Verfahren Ödön von Horváths'. Wirkendes Wort, 25 (1975), pp.197-214.

Rotermund, Erwin. 'Zur Erneuerung des Volksstückes in der Welmarer Republik: Zuckmayer und Horváth'. In: Traugott Krischke/Dieter Hildebrandt (Hg.). Uber Ödön von Horváth. Frankfurt/M., Suhrkamp, 1972 (es 584), pp.18-45. 
Schmidt-Dengler, Wendelin. 'Ödön von Horváths Geschlchten aus dem Wiener Wald und der triviale Wiener Roman der zwanziger Jahre'. In: Traugott Krischke (Hg.). Ödön von Horváth. Frankfurt/M., Suhrkamp, 1981 (st 2005), pp.57-66.

Schnelder, Hansförg. 'Der Kampf zwischen Individuum und Gesellschaft'. In: Traugott Krischke/Dieter Hildebrandt (Hg.). ther Ödön von Horváth. Frankfurt/M., Suhrkamp, 1972 (es 584), pp.59-70.

Schröder, Jürgen. 'Das Spätwerk Ödön von Horváths'. In: Traugott Krischke (Hg.). Ödön von Horváth. Frankfurt/M., Suhrkamp, 1981 (st 2005), pp.125-155.

Stenberg, Peter. 'The last of the magicians. Horváth's Zauberkönig and his ancestors!. German Life and Letters 28 (1974/75), pp.395-405.

Stoljar, Margaret Mahoney. 'The drama as metaphor: meaning and structure in Ödön von Horváth's Volksstiicke'. AUMLA 48. (1977), pp.333-350.

Strelka, Joseph. Brecht, Horváth, Dürrenmatt. Wege und Abwege des modernen Dramas. Wien, Hannover, Bern, Forum, 1962.

von Wiese, Benno. 'Ödön von Horváth'. In: Traugott Krischke (Hg.)... Ödön von Horváth. Frankfurt/M., Suhrkamp, 1981 (st 2005), pp.7-45. Reprinted from Benno von Wiese (Hg.). Deutsche Dichter der Moderne. Berlin, Erich Schmidt, 1975.

Walder, Martin. Die Unelgentlichkelt des Bewubtseins. Zur Dramaturgle ödōn von Horváths. Bonn, Bouvier, 1974.

Winston, Krishna. Horváth Studies. Close Réadings of Six Plays (1926-31). Bern, Frankfurt, Las Vegas, Peter Lang, 1977. 


\section{2) Comedy Theory}

\section{1) Collectlons of Essays}

Das Komlsche. Hg. Wolfgang Prelsendanz/Rainer Warning. München, Wilhelm Fink, 1976. (Poetik und Hermeneutik VII).

Die deutsche Komödle im 20. Jahrhundert. Hg. Wolfgang Paulsen. Heldelberg, Lothar St1ehm Verlag, 1976. (6. Amherster Kolloquium 1972).

Die deutsche Komödie Vom Mittelalter bis zur Gegenwart. Hg. Walter Hinck. Düsseldorf, August Bagel Verlag, 1977.

Wesen und Formen des Komischen. Hg. Klaus Berghahn/Reinhold Grimm. Darmstadt, Wissenschaftllche Buchgesellschaft, 1973. (Wege der Forschung LXII).

\section{1) Articles and Books}

Arntzen, Helmut. 'Wirklichkelt als Kolportage. Zu drel Komödien von Georg KaIser und Robert Mus1l'. DVjs, 36 (1962), pp.544-561.

Arntzen, Helmut. Die ernste Komödie. Das deutsche Lustsplel von Lessing bis Klelst. München, Nymphenburger Verlagshandlung, 1968.

Arntzen, Helmut. 'Komödie und episches Theater'. In: Wesen und Formen des Komischen. Hg. Klaus Berghahn/Reinhold Grimm. Darmstadt, Wissenschaft 1 lche Buchgeselischaft, 1973. (Wege der Forschung LXII), pp.441-455.

Bayerdörfer, Hans-Peter. 'Non olet - altes Thema und neues Sujet: Zur Entwicklung der Konversationskomödie zwlschen Bestauration und Jahrhundertwende'. Euphorion, 67 (1973), pp.323-358.

Bergson, Henri. Le Rire. Essai sur la signification du comique. Paris, 1922.

Catholy, Eckehard. 'Komische Person und dramatische Wirklichke1t. Ein Versuch zur Typologle des Dramas'. Festschrift Helmut de Boor. Tübingen, Nlemeyer, 1966, pp.193-208. 
Catholy, Eckehard. 'Posse'. In: Reallexikon der deutschen Literaturgeschichte. Bd.3. Hg. Werner Kohlschmidt und Wolfgang Mohr. Berlin, Walter de Gruyter, 1977. 2.Auflage.

Daunicht, Richard/Kohlschmidt, Werner/Mohr, Wolfgang. 'Lustspiel'. In: Reallexikon der deutschen Literaturgeschichte $-\mathrm{Bd} .2$. $\overline{\mathrm{Hg}}$. Werner $\overline{\mathrm{Koh}}$.schmidt und Wolfgang Mohr. Berlin, Walter de Gruyter, 1965. 2.Auflage.

Doppler, Alfred. 'Das Konversationsstïck be1 Arthur Schnitzler und Hugo von Hofmannsthal'. In: Sprachthematik in der österrelchischen Literatur des 20 . Jahrhunderts. Hg. vom InstItut für ÓsterreIchkunde, Wien, HIrt, 1974, pp.69-82.

Duckworth, G.E. The Nature of Roman Comedy: A Study in Popular Entertainment. Princeton, Princeton Un1v. Press, 1952.

Eder, Klaus. Ant1ke Komödie. München, dtv., 1974. (Friedrichs Dramatiker des welttheaters 6830 ).

Frye, Northrop. Anatomy of Critic1sm: 4 Essays. Princeton, Princeton UnIv. Press, 1957.

Fuhrmann, Manfred. 'Lizenzen und Tabus des Lachens - Zur sozlalen Grammatik der hellenistisch-römischen Komödie'. In: Das Komlsche. Hg. Wolfgang Prelsendanz/Rainer Warning. Minchen, WIIhelm Fink, 1976. (Poetik und Hermeneutik VII), pp. 65-101.

Giese, Peter Christian. Das Gesellschaft-Komische: Zu Komik und Komödie am Betspiel der Stïcke und Bearbeitungen Brechts. Stuttgart, J. B. Metzlersche Verlagsbuchhandlung, 1974 .

Grimm, Reinhold. 'Neuer Humor? Komödienproduktion zwischen 1918 und 1933'. In: Die deutsche Komödie Im 20. Jahrhundert. Hg. Wolfgang Paulsen. Heidelberg, Lothar Stiehm Verlag, 1976. (6. Amherster Kolloqulum 1972), pp.107-133.

Grimm, Reinhold. 'Komik und Verfremdung'. In: Wesen und Formen des Komischen. Hg. Klaus Berghahn/Reinhold Grimm. Darmstadt, Wissenschaftliche Buchgesellschaft, 1973. (Wege der Forschung LXII), pp.253-271.

Guthke, Karl S. Geschichte und Poetik der deutschen Trag1komödie. Göttingen, Vandenhoeck \& Ruprecht, 1961. 
Halda, Peter. Komödie um 1900. Wandlungen eines Gattungsschemas von Hauptmann bis Sternheim. München, Wilhelm Fink, 1973. (Kritische Informationen 7).

HeIn, Jürgen (Hg.). Theater und Gesellschaft. Das Volksstlick im 19. und 20. Jahrhundert. Düsseldorf, Bertelsmann Un1versitätsverlag, 1973 .

Hein, Jürgen. 'Volksstücke'. In: Welmarer Republik-Drittes Reich-Avantgardismus-Partellichkelt-Ex11. Vol.9 of Deutsche Literatur: EIne Sozlalgeschichte. Hg. Alexander von Bormann/Horst Albert Glaser. Reinbek bel Hamburg, Rowohlt, 1983, pp.264-272.

Hinck, Walter. Das deutsche Lustspiel des 17. und 18. Jahrhunderts und die Italienische Komödie. Stuttgart, J.B. Metzlersche Verlagsbuchhandlung, 1965.

Hinck, Walter. Das moderne Drama in Deutschland. Vom expressionistischen zum dokumentarischen Theater. Göttingen, Vandenhoeck \&uprecht, 1973.

Hinck, Walter. Vom Ausgang der Komödie. Exemplarische Lustspielschlüsse in der europäischen Literatur. Opladen, Westdeutscher Verlag, 1977 . (Rheinfch-Westfälische Akademie der Wissenschaften Vorträge G221).

Hinck, Walter. 'Einleltung zur Theorle des Komischen und der Komödie'. In: Dle deutsche Komödie Vom Mittelalter bis zur Gegenwart. Hg. Walter Hinck. Dissseldorf, August Bagel Verlag, 1977, pp.11-31.

Holl, Karl. Geschichte des deutschen Lustspiels. Leipzig, J.J. Weber, 1923.

Howarth, W.D. Comic Drama. London, Methuen, 1978.

Kayser, Wolfgang. Das sprachliche Kunstwerk. Elne Einführung in die Literaturwissenschaft. Minchen, Berm, Francke, 1948. 15. Auflage.

Kindermann, Heinz. 'Grundformen des komischen Theaters'. In: Wesen und Formen des Komischen. Hg. Klaus Berghahn/Reinhold Grimm. Darmstadt, Wissenschaft tiche Buchgesellschaft, 1973. (Wege der Forschung LXII), pp.93-126. 
Klotz, Volker. Bürgerllohes Lachtheater: Komödie, Posse, Schwank, Operette. München, dtv, 1980.

Kluckhohn, Paul. 'Die Arten des Dramas'. DVjs 19 (1941), pp. $241-268$.

Langer, Suzanne K. Feeling and Form. A Theory of art developed from Philosophy in a New Key. New York, Charles Scribner's Sons, 1953.

Magill, C.P. 'The Comic Muse in Germany'. Moderm Languages 31 $(1949 / 50)$, pp.18-24.

Mag111, C.P. 'Der Gross-Cophta and the problem of German Comedy'. In: German Studies. Presented to W.H. Bruford.

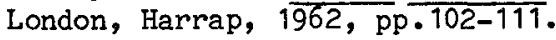

Malsch, Wilfrled. 'Theoretische Aspekte der modermen Komödie'. In: Die deutsche Komöd Ie Im 20. Jahrhundert. $\mathrm{Hg}$. Wolfgang Paulsen. Heidelberg, Lothar Stiehm Verlag, 1976. (6. Amherster Kolloquium 1972), pp.27-43.

Martini, Fritz. Lustspiele und das Lustspiel. Stuttgart, Ernst Klett, 1974.

Müller, Gerd. Das Volksstück von Raimund bis Kroetz. Die Gattung in Einzelanalysen. München, R. Oldenbourg Verlag, 1979.

Miiller-Seldel, Walter. 'Dramatische Gattungen'. In: Das Fischer Lexikon Literatur II Erster Te11. Hg. Wolf-Hartmut Friedrich/Walther Killy. Frankfurt/M., Fischer, 1965, pp.162-184.

Olsen, Elder. The Theory of Comedy. Bloomington, Indiana Univ. Press, $196 \overline{8}$

Prang, Helmut. Geschichte des Lustspiels. Stuttgart, Kröner, 1968.

Rommel, Otto. 'Die wissenschaftlichen Bemühungen um die Analyse des Komischen'. DV js 21 (1943), pp.252-286.

Rommel, Otto. 'Komik und Lustspieltheorie'. DVjs 21 (1943), pp.252-286. 
Scheck, Ulrich. Parodie und Eigenständigkelt in Nestroys 'Judith und Holofernes': Ein Vergleich mit Hebbels 'Judith'. Bern, Frankfurt/M., Peter Lang, 1981.

Schmidt-Dengler, Wendelin. 'Das Kontrastschema Stadt-Land in der Alt-Wiener Volkskomödie'. In: Jürgen Hein (Hg.). Theater und Gesellschaft. Das Volksstïck im 19. und 20. Jahrhundert. Disseldorf, Bertelsmann Universitätsverlag, $\overline{1973}$, pp.57-68.

Schmidt-Dengler, Wendelin. 'Die Unbedeutenden werden bedeutend. Anmerkungen zum Volksstiuck nach Nestroys Tod: Kaiser, Anzengruber und Morre'. In: Die Andere Welt: Aspekte der österrelchischen Literatur des 19. und 20. Jahrhunderts. Hg. Kurt Bartsch et. al. Berm, Müchen, Francke, 1979, pp.133-146.

Schürer, Ernst. 'DIe nachexpressionistische Komödie'. In: Die deutsche Literatur der Welmarer Republik. Hg. Wolfgang Bothe. Stuttgart, Reclam, 1974, pp. 47-76.

Steffen, Hans (Hg.) Das deutsche Lustspłel. 2 vols. Göttingen, Vandenhoeck \& Ruprecht, $1 \overline{969 .}$ (Kleine Vandenhoeck Reihe 277).

Tschižewskij, Dimitrij. 'Satire oder Groteske'. In: Das Komische. Hg. Wolfgang Preisendanz/Rainer Warning. Minchen, Wilhelm Fink, 1976. (Poetik und Hermeneutlk VII), pp.269-278.

Warning, Rainer. 'Elemente elner Pragmasemlotik der Komödie'. In: Das Komische. Hg. Wolfgang Prelsendanz/Rainer Warning. Minchen, Wilhelm Fink, 1976. (Poetik und Hermeneut1k VII), pp.279-333.

\section{3) Other Works Consulted}

Arntzen, Helmut. Mus1l-Kommentar. München, Winkler, 1980.

Aufricht, Ernst Josef. Erzähle, damit du dein Recht erweist. Berlin, Propyläen, 1966.

Bartsch, Kurt et. al. (Hg) Dle Andere Welt: Aspekte der österre1chischen Literatur des 19. und 20. Jahrhunderts: Bern, Mínchen, Francke, $197 \overline{9}$. 
Baur, Uwe. Dorfgeschichte. Zur Entstehung und gesellschaftlichen Funktion einer literarischen Gattung im Vormärz. München, Wilhelm Fink Verlag, 1978.

Bausinger, Hermann. Formen der 'Volkspoesle'. Berlin, Erich Schmidt, 1980. 2. Auflage.

Benkard, Ernst. Das ewige Antlitz: Eine Sammlung von Totenmasken. Berlin, Frankfurter Verlags-Anstalt, 1927.

Beitl, Richard. Wörterbuch der deutschen Volkskunde. Stuttgart, Kröner, 1974. 3. Auflage.

Bloch, Ernst. Das Prinzip Hoffnung. Frankfurt/M., Suhrkamp, 1959.

Bloch, Ernst. Erbschaft dieser Zeit. Frankfurt/M., Suhrkamp, 1962.

Bloch, Ernst. Gelst der Utople. Unveränderter Nachdruck der bearbeiteten Neuauflage der zweiten Fassung von 1923. Frankfurt/M., Suhrkamp, 1973.

Burckhardt, Jacob. History of Greek Culture. Trans. by Palmer Hitty. London, Constable, 1963.

Denkler, Horst. 'Sache und St1l. Die Theorle der "Neuen Sachlichkelt" und 1hre Auswirkungen auf Kunst und Dichtung'. Wirkendes Wort 18 (1968), pp.167-185.

Dietrich, Margaret/KIndermann Heinz. Dichtung aus Österreich: Drama. Wien, München, Österreichischer Bundesver Iag, 1966.

Donnenberg, Josef. 'Das Thema HEIMAT in der Gegenwartsliteratur und Anzengruber als Schlüsselfigur der Tradition der Helmatliteratur'. In: Traditionen in der neueren österreichischen Literatur. Hg. Friedbert Aspetsberger. Wien, Osterrrelchischer Bundesverlag, 1980, pp.67-82.

Diurrenmatt, Friedrich. Theaterprobleme. In: Friedrich Dürenmatt. Theater-Schriften und Reden. Hg. Elisabeth Brock-Sulzer. Zürich, Arche, 1966, pp.92-131.

Frenzel, Elisabeth. Stoffe der Weltliteratur. Stuttgart, Kröner, 1970. 3. Auflage. (Kröners Taschenausgabe 300). 
Frenzel, Elisabeth. Motlve der Weltliteratur. Stuttgart, Kröner, 1976. (Kröners Taschenausgabe 301).

Freud, Sigmund. Die Traumdeutung. Frankfurt/M., Fischer Taschenbuch Verlag, 1977.

Freud, S1gmund. Werkausgabe in zwe1 Bänden. Hg. Anna Freud/Ilse Grubrich Simitis. Frankurt/M., Fischer, 1978.

Gnïg, Hiltrud (Hg.). Literarische Utopie-Entwïfe. Frankfurt/M., Suhrkamp, 1982. (st 2012).

Grelner, Martin. 'Helmatkunst'. In: Reallexikon der deutschen Literaturgeschichte. Bd.1. Hg. Wermer Kohlschmidt und Wolfgang Mohr. Berlin, Walter de Gruyter, 1958. 2.Auflage.

Grimm, Reinhold/Hermand, Jost (Hg.). Deutsches utopisches Denken Im 20. Jahrhundert. Stuttgart, Berlin, Köln, Mainz, W. KohIhammer, 1974. (Urban-Taschenblicher 864).

Grimm, Reinhold. 'Pyramide und Karussell'. In: Reinhold Grimm. strukturen. Göttıngen, Sachse \& Pohl, 1963, pp.8-43.

Grosz, George. Ecce Homo. New York, Grove Press, 1966.

Hegel, Georg Wilhlem Friedrich. Ästhetik. Mit elner Einführung von Georg Lukács. 2 vols. Frankfurt/M., Europälsche Verlagsanstalt, n.d.

Heldegger, Martin. Sein und Zelt. Erste Hälfte. 4. Auflage. Halle, Nlemeyer, 1935 .

Hempfer, Klaus W. Gattungstheorle. Mïnchen, Wilhelm Fink, 1973. (UTB 133).

Herd, M.J. Role-playing in the Plays of Max Frisch and Jean Anouilh. Ph.D. Dunedin, UnIv. of otago, 1977.

Hermand, Jost. 'Einheit in der Vielhelt? Zur Geschichte des Begriffs "Neue Sachlichkelt"'. In: Das Iiterarische Leben In der Welmarer Republik. Hg. Keith Bullivant. Kronberg/Ts., Scriptor Verlag, 1978, pp.71-88.

Holdheim, Wolfgang W. Der Justizirrtum als Ifterarische Problematik. Verglelchende Analyse elnes erzählerischen Themas. Berlin, Walter de Gruyter, 1969. 
Innes, Christopher. Moderm German Drama. A Study in Form. Cambridge, Cambridge Univ. Press, 1979.

Irmscher, Johannes. Das groBe Lexikon der Antike. München, Heyne, 1963.

Jaron, Norbert. Das demokratische Zeittheater der späten 20er Jahre. Untersucht am Beispiel der stücke gegen die Todesstrafe. Elne Rezeptionsanalyse. Frankfurt/M., Bem, Peter Lang, 1981.

Kayser, Wolfgang. The Grotesque In Art and L1terature. Trans. by Ulrich Welsstein. New York, McGraw Hill, 1966.

Kerr, Alfred. Theaterkritiken. Hg. Jürgen Behrens. Stuttgart, Reclam, 1971.

Killy, Walther, Deutscher Kitsch. Göttingen, Vandenhoeck \& Ruprecht, $196 \overline{2 .}$

Klotz, Volker. Geschlossene und offene Form im Drama. München, Carl Hanser Verlag, 1969.

Koebner, Thomas. 'Das Drama der Neuen Sachlichke1t und dle Krise des Liberalismus'. In: Die deutsche Literatur der Weimarer Republik. Hg. Wolfgang Rothe. Stuttgart, Reclam, 1974, pp.19-46.

Kracauer, Siegfried. Die Angestellten. Frankfurt/M., Suhrkamp, 1971.

Kracauer, Siegfried. From Caligari to Hitler. A psychological history of German film. Princeton, Princeton Univ. Press, 1947.

La Place, Roselyn. 'Des théâtres d'enfants au XVIII siècle'. revue d'histoire du théâtre 1 (1980), pp.21-31.

Lethen, Helmut. Neue Sachlichke1t 1924-1932. Studien zur Literatur des "Weiben Sozialismus". Stuttgart, J.B. Metzlersche Verlagsbuchhandlung, 1970.

Mennemeler, Franz Norbert/Trapp Frithjof. Deutsche. Exildramatik 1933 bis 1950. München, Wilhelm Fink, 1980 . 
Pfister, Manfred. Das Drama. Minchen, Wilhelm Fink, 1977. (UTB $580)$.

Phillpott, Beatrice. Mermaids. New York, Ballantine, 1980.

Relch, Wilhelm. Mass Psychology of Fascism. Trans. Vincent Carfagno. London, Penguin, 1970 .

Rossbacher, Karlheinz. 'Programm und Roman der Helmatkunstbewegung - Mögllchkeiten sozlalgeschichtlicher und sozlologischer Analyse.' In: Deutsche Literatur der Jahrhundertwende. Hg. Viktor Żmegač. Kronberg/Ts., Scriptor Verlag, 1981, pp.123-144.

Rïilger, Horst. (Hg.) Kleines literarisches Lexikon: Sachbegrtffe 3. Bern, München, Francke, 1966.

Ruhle, Gïnter. Theater für die Republik 1917-1933. Im Spiegel der Kritik. Frankfurt/M., Fischer, 1967 .

Ruihle, Günter.
Republik $\frac{\text { Zeit }}{\text { zur }} \frac{\text { und }}{\text { Diktatur. }} \frac{\text { Theater }}{\text { Frankfurt/M. }} \frac{1925-1933 .}{\text { Bd.3: }} \frac{\text { Von }}{\text { der }}$ U1Istein, $1 \overline{972}$.

Rühle, Günter, Materlalien zum Leben und Schrelben der Marleluise Fle1Ber. Frankfurt/M., Suhrkamp, 1973.

Ruprecht, Erich/Bänsch, Dieter (Hg.). Literarische Manlfeste der Jahrhundertwende 1890-1910. Stuttgart, J.B. Metzlersche Verlagsbuchhand Iung, 1970 .

Schabert, Ina. (Hg.) Shakespeare-Handbuch. Stuttgart, Kröner, 1978.

Schanze, Helmut. Drama im bürgérlichen Zeltalter 1850-1890. Frankfurt/M., Vittorio Klostermann, 1973.

Schmidt, Adalbert. 'Ludwig Anzengruber Das vierte Gebot'. In: Das österrelch1sche Volksstück. Hg. vom Inst1tut für Osterrelchkunde. Wien, Ferdinand Hirt, 1971, pp.59-76.

Sengle, Frledrich. 'Wunschbild Land und Schreckbild Stadt: Zu einem zentralen Thema der neueren deutschen Literatur'. Studium Generale 16,10 (1963), pp.619-631. 
Sinor, Denis. History of Hungary. London, Allen and Unwin, 1959.

Sontheimer, Kurt. Antidemokratisches Denken in der Welmarer Republik. München, dtv., 1968. (Wissenschaftliche Relhe).

Steiner, George. The Death of Tragedy. London, Faber, 1961.

Staiger, Emil. Grundbegriffe der Poetik. Zürich, Atlantis, 1968.

Szond1, Peter. Theorie des modernen Dramas 1880-1950. In: Peter Szondi. Schriften I. Frankfurt/M., Suhrkamp, 1978. (stw 219).

Völker, Klaus. Wedekind, Velber, Frledrich Verlag, 1965. (Friedrichs Dramatiker des Welttheaters 7).

Wächter, Hans-Christof. Theater in Exil. Sozlalgeschichte des deutschen Ex1ltheaters 1933-1945. München, Hanser, 1973.

Walter, Hans-Albert. Deutsche Exilliteratur 1933-1950. Bd.2 Asylpraxis und Lebensbedingung in Europa. Darmstadt und Neuwled, Luchterhand, 1972.

Wilpert, Gero von. Sachwörterbuch der Literatur. Stuttgart, Kröner, 1969. 5. Auflage.

Zuckmayer, Carl. Als wär's eln Stück von mir. Frankfurt/M., Fischer Taschenbuch, 1966. 